\title{
INBREEDING IN DARWIN'S MEDIUM GROUND FINCHES
}

(GEOSPIZA FORTIS)

\author{
H. LISLE GiBBS ${ }^{1.2}$ \\ Museum of Zoology and Department of Biology, The University of Michigan, \\ Ann Arbor, MI 48109-1079 \\ AND \\ Peter R. Grant \\ Department of Biology, Princeton University, Princeton, NJ 08544-1008
}

\begin{abstract}
We studied the frequency and causes of inbreeding and its effect on reproductive success in a population of Darwin's Medium Ground Finches (Geospiza fortis) on Isla Daphne Major, Galápagos, during four breeding seasons (1981, 1983, 1984, and 1987). Pedigree analysis showed that levels of inbreeding were low but comparable with those observed in other passerine birds. For pairs with at least half of their grandparents known, approximately $20 \%$ of all pairings were between detectably related birds. The frequency of pairings between closely related birds (coefficient of kinship $[\phi] \geq 0.250$ ) among all pairs was $0.6 \%$. We detected no effect of inbreeding on reproductive success, although sample sizes were small. The observed reproductive output of related pairs was not significantly different from the output of unrelated pairs, and there was no correlation between a pair's kinship coefficient and an estimate of the potential magnitude of inbreeding depression. Comparisons with a study of Great Tits (Parus major) by van Noordwijk and Scharloo (1981) suggest that, even if present, the fitness costs of inbreeding in this population of $G$. fortis would be low. Observed levels of inbreeding in each breeding episode were accurately predicted by simulations of random mating in which relatedness had no influence on pairing between individuals. This result suggests that levels of inbreeding in this population are determined more by demographic factors than by behavioral avoidance of mating with kin.
\end{abstract}

Received June 1, 1988. Accepted March 24, 1989

It has often been argued that inbreeding has important evolutionary consequences in vertebrate populations (Mayr, 1963; Wright, 1969). For example, levels of inbreeding can influence the overall amount of genetic variation present within populations and the degree of kinship among individuals, while the negative effects of inbreeding on fitness may play an important role in the evolution of a wide variety of behaviors, including patterns of mate choice (Bateson, 1978) and sex-specific patterns of dispersal within species (Greenwood and Harvey, 1982).

Inbreeding is generally defined as mating between related individuals, with kinship judged from some arbitrarily defined reference population or pedigree (see Shields [1982] and Ralls et al. [1986] for recent discussions). Despite its potential importance, inbreeding has been rarely studied in natural populations of birds, mainly because

\footnotetext{
'Present address: Department of Biology, Queen's University, Kingston, ON K7L 3N6, Canada.

${ }^{2}$ To whom all correspondence should be addressed.
}

direct estimates of inbreeding and the mechanisms responsible can only be obtained from long-term studies of marked individuals (e.g., studies on the Great Tit [Parus major]: van Noordwijk and Scharloo [1981]; van Noordwijk et al. [1985]; van Tienderen and van Noordwijk [1988]). However, the following key questions about inbreeding remain largely unanswered. 1) How frequently does inbreeding occur? 2) What is the effect, if any, of inbreeding on reproductive success? 3) What is the relative importance of factors such as dispersal, population structure, and behavioral avoidance of mating with kin in determining levels of inbreeding?

Population-level estimates of inbreeding, inferred from pedigrees, have been calculated for several bird species (Bulmer, 1973; Greenwood et al., 1978; van Noordwijk and Scharloo, 1981; Payne et al., 1985; Ralls et al., 1986; Rowley et al., 1986; Craig and Jamieson, 1988). Most studies, however, have only estimated levels of inbreeding from matings between close relatives (parents and offspring or siblings breeding with 
each other). As Templeton (1987) suggests, and van Noordwijk and Scharloo's (1981) study of inbreeding in Great Tits (Parus major) demonstrates, matings between more distant relatives can make an important contribution to observed levels of inbreeding and should be incorporated into estimates of the overall frequency of inbreeding.

Studies that examine the fitness consequences of inbreeding in wild birds are even rarer. Inbreeding in domesticated or captive birds generally has a negative effect on components of fitness such as egg hatchability and production (Shoffner, 1948; Lerner, 1954; Sittmann et al., 1966; but see Daniell and Murray [1986]). However, the effects of inbreeding on reproductive success in wild birds vary. Hatching success is negatively correlated with pair relatedness in Great Tits (van Noordwijk and Scharloo, 1981; see also Greenwood et al. [1978]). In contrast, highly inbred pairs of Splendid Fairy Wrens ( $\mathrm{Mal}$ urus splendens) show no decrease in success compared with less inbred birds (Payne et al., 1985; Rowley et al., 1986). Clearly, more studies are needed to determine whether inbreeding usually is associated with a fitness cost.

Moreover, the relative importance of behavioral avoidance and population structure in generating observed levels of inbreeding in wild bird populations is largely unknown. There is indirect evidence, based on dispersal patterns, that individuals in some species avoid mating with close kin (Koenig and Pitelka, 1979). Ralls et al. (1986) suggest that the low percentage of matings observed between close kin in a number of populations implies that behavioral avoidance of mating with close relatives is common among birds. These studies, however, did not attempt to compare observed levels of inbreeding with those expected if birds were mating at random with respect to relatedness. This comparison has only been attempted for a single population of Great Tits (van Noordwijk et al., 1985; van Tienderen and van Noordwijk, 1988), and the results demonstrated that pairing was random with respect to relatedness. This finding suggests that the level of inbreeding in this population is determined mainly by aspects of population structure such as pop- ulation size, degree of population subdivision, and variance in reproductive success among individuals.

The purpose of our study was to examine the level, causes, and fitness consequences of inbreeding in a population of Darwin's Medium Ground Finches (Geospiza fortis) on Isla Daphne Major, Galápagos. This population has been intensively studied since 1976 (see Boag and Grant, 1984; Price, 1985; Gibbs and Grant, 1987a), with the result that practically all adults in the population have been individually marked since 1978. Thus, detailed pedigrees and life-history information are available for many individuals. The specific goals of the study were to 1) measure the level of inbreeding, using a pedigree analysis, 2) determine whether there was an effect of pair-relatedness on reproductive success, and 3 ) identify the factors responsible for the observed level of inbreeding in the population.

\section{Materials ANd Methods Finch Populations}

The population of Darwin's Medium Ground Finches (Geospiza fortis) has been studied intensively on Isla Daphne Major, Galápagos, for the first 6-9 months of each year from 1976 until the present (Boag and Grant, 1984; Price, 1985; Gibbs and Grant, $1987 a$ ). Isla Daphne Major (henceforth, "Daphne") is a small island (<40 ha) situated in the middle of the Galápagos archipelago. A description of its geological and floristic characteristics is given in Boag and Grant (1984). Over $95 \%$ of all $G$. fortis individuals in the population have been banded with a metal band and a unique combination of three colored plastic bands since 1978. Due to the demography of the population, the 1981 breeding season was the first in which matings between related birds could be detected. Owing to annual variation in rainfall, birds do not breed in every year. Here, we present data on inbreeding in four years $(1981,1983,1984$, and 1987) when enough reproduction occurred for us to identify breeding pairs reliably and to obtain information on their reproductive success.

Finches breed in response to rain, which can fall any time during December-July. 
During each of the four breeding seasons reported here, all nests were marked, and the parents were identified. Nest contents were regularly checked to determine clutch size, hatching, and fledging success. We are confident that more than $95 \%$ of all nests were found, because repeated systematic searches of all potential nest sites were conducted and because fledglings without bands were rarely seen. Young birds that were alive in January of the year following the season of their birth were recorded and identified during repeated and standardized visual censuses and other observations (Boag and Grant, 1984; Gibbs and Grant, 1987b).

\section{Inbreeding Analyses}

Inbreeding is defined here as any mating between detectably related individuals (see van Noordwijk and Scharloo, 1981). The degree of relatedness between members of a pair is described by their coefficient of kinship $(\phi)$, which is the probability that an allele chosen randomly from either individual would be identical by descent (Malécot, 1948). The coefficient of kinship of a pair is equal, by definition, to the coefficient of inbreeding of their offspring. Kinship coefficients for all pairs in each breeding season were calculated from pedigrees with the SAS Supplemental Library Program INBREED (Reinhardt, 1979). We assumed that pairs with no detected common ancestors had a kinship coefficient of zero. Clearly, the observed level of kinship is a minimum estimate, because common ancestors for many birds were not known. The depth of the pedigree for the population varied considerably through time; in 1981, the minimum detectable level of kinship was $\phi=0.125$. By 1987 , this value had decreased to 0.008 .

\section{Expected Levels of Inbreeding}

To determine whether the frequency of inbreeding is greater or less than expected by chance, we followed the empirically based approach of van Noordwijk et al. (1985) and van Tiederen and van Noordwijk (1988) and used computer simulations of pairing between individuals to determine whether relatedness influenced mate choice and, hence, levels of inbreeding. This approach has three advantages over the less direct ways of estimating expected levels of inbreeding that are based on calculations involving effective population size (see Crow and Kimura, 1970): 1) the demographic data needed to estimate $N_{\mathrm{e}}$ are extensive and difficult to obtain for natural populations; 2) changes in our knowledge of ancestry in the population, which strongly influence detected levels of inbreeding, can be incorporated into our estimates of expected levels of inbreeding; and 3) with simulations, we were able to generate a distribution of expected values of kinship under random mating and, hence, to obtain a plausible estimate of the variance of the distribution.

We compared observed and random patterns of pairing during the first episode of breeding in each of four years: 1981, 1983, 1984 , and 1987. We did the same for the middle of the 1983 season (April), when large numbers of young birds, born earlier in the year, began breeding (see Gibbs et al., 1984; Gibbs and Grant, 1987a). We considered only the first episode of mating in most years, because the formation of most new pairs takes place at the beginning of each breeding season. For each breeding episode, we used the following procedure to generate a frequency distribution of expected levels of kinship if pairing were random with respect to relatedness.

1) To determine which birds could potentially pair at the start of a year, we identified all reproductively active females and males on the island from census data. During 1981-1984, breeding females were identified as all females that laid eggs within the first 30 days of the breeding season. In 1987 , the sex ratio was slightly female-biased; hence, all potentially reproductive females did not breed immediately. In this year, female breeders were identified as birds that had reproduced in previous years. In all years, males were identified as birds that actively defended territories.

2) We then excluded from this group those males and females that had bred with the same mate in the previous breeding season, on the assumption that these individuals would not be available for remating.

3) We then paired each member of the limiting sex (females in all years except 
1987) with a single randomly chosen member of the opposite sex and calculated $\phi$ for each randomly formed pair. The mean level of inbreeding for the entire round of simulated mating was calculated as the mean $\phi$ value for all pairs (randomly formed pairs and long-term pairs combined). Both randomly and previously formed pairs were combined in order to make this estimated value directly comparable with the observed value (see step 5).

4) This procedure was then repeated 1,000 times to generate an expected distribution of random pairings for each breeding episode.

5) Observed levels of inbreeding were calculated as the mean $\phi$ value for first pairings of all breeding females (19811984 ) or breeding males (1987) within a given reproductive episode.

Our simulation of random mating for this population makes several assumptions that are somewhat unrealistic about the pool of potential mates and their pairing behavior. First, we assumed that patterns of dispersal do not influence the probability of each unmated bird pairing with each unmated member of the opposite sex. Van Tiederen and van Noordwijk (1988) discuss how the dispersal patterns of individuals can influence levels of inbreeding in a natural population. Second, we assumed that pairing occurs simultaneously among all unpaired birds during a breeding episode, i.e., that the population is not subdivided as a result of different individuals breeding at different times.

These assumptions, however, may not deviate very strongly from the observed behavior of the birds. First, the small size of the island (maximum N-S diameter $=743$ $\mathrm{m}$; maximum $\mathrm{E}-\mathrm{W}$ diameter $=560 \mathrm{~m}$ ), and the movement of juvenile and nonbreeding birds over much of the island (Gibbs and Grant, unpubl.), suggest that mate choice is probably not constrained by dispersal opportunities. Natal dispersal probably occurs over the entire island; however, judging from very limited data, females disperse farther than males, as has been found in some other passerine species (Greenwood and Harvey, 1982). For example, for birds born in 1983 that bred in the same year, the median dispersal distance was greater for females than for males (females: median distance $=263$ $\mathrm{m}$, range $=37-634 \mathrm{~m}, N=104$; males: median distance $=190 \mathrm{~m}$; range $=18-525 \mathrm{~m}$, $N=61$; Mann Whitney $U$ test, $P<0.05$ ). This difference between the sexes violates the "equal chance" assumption, because the observed distances suggest that females can potentially disperse over the entire island while males are more restricted in their movements. It may be unimportant, however, since females (the wide-ranging sex) were also the "choosing" sex in all years except 1987, due to the male-biased sex ratio on the island during 1981-1984 (Price, 1984; Gibbs and Grant, unpubl.). The second assumption, that pairing occurs simultaneously, is generally upheld in our population; pairing at the start of a breeding season occurs rapidly, usually over a 1-2week period (Price, 1984).

\section{Success of Related Pairs}

We evaluated the effect of inbreeding on eight related measures of reproductive success at different stages of the life cycle: clutch size, number of young that hatched, number of young that fledged, number of young that survived to the following year, percentage of eggs that hatched, percentage of eggs that fledged, percentage of hatched young that fledged, and percentage of fledged young that survived. We compared the observed success of related pairs with an estimate of their expected performance had they been unrelated. This estimate was calculated from a sample of pairs from the same season that were not known to be related; pairs were matched for factors known to have a proximate effect on reproductive success, such as pair age (Gibbs et al., 1984) and seasonal changes in reproductive success (Boag and Grant, 1984; Price, 1985; Gibbs and Grant, 1987a). Greenwood et al. (1978) and Rowley et al. (1986) adopted a similar approach in their studies of inbreeding in other birds. Some undetected closely related pairs were probably included in the nonrelated group, particularly in the early years of the study, when the pedigree for the population was shallow. To reduce these pairs to a minimum, we used only pairs from age-month classes that had the greatest number of 
TABLE 1. Occurrence of detected inbreeding in G. fortis on Isla Daphne Major from 1981 to 1987.

\begin{tabular}{|c|c|c|c|c|c|c|}
\hline \multirow[b]{2}{*}{ Pair type } & \multirow[b]{2}{*}{$\begin{array}{l}\text { Number } \\
\text { of pairs }\end{array}$} & \multicolumn{2}{|c|}{ Detected inbreeding } & \multicolumn{2}{|c|}{$\begin{array}{c}\text { Detected close } \\
\text { inbreeding } \\
(\phi \geq 0.125)\end{array}$} & \multirow[b]{2}{*}{$\begin{array}{c}\text { Mean } \\
\text { inbreeding ( } \phi)\end{array}$} \\
\hline & & $\begin{array}{l}\text { Number } \\
\text { of pairs }\end{array}$ & $\%$ & $\begin{array}{l}\text { Number } \\
\text { of pairs }\end{array}$ & $\%$ & \\
\hline Unique pairs (male and female identified) & 583 & 27 & 4.6 & 8 & 1.4 & 0.00394 \\
\hline Parents of male and female known & 345 & 26 & 7.5 & 7 & 2.0 & 0.00592 \\
\hline Parents and at least half of the grandparents known & 70 & 17 & 24.3 & 0 & 0 & 0.0120 \\
\hline All grandparents known & 10 & 2 & 20.0 & 0 & 0 & 0.0110 \\
\hline
\end{tabular}

known ancestors and were represented by at least 15 pairs. Approximately half of the related pairs bred more than once within a year or in different breeding seasons. Since the performances of these birds during repeated breeding attempts were not independent, we averaged the observed measures of success from two or more attempts, did the same for the expected measures of success, and thereby obtained single values for the expected and observed performances of each related pair over the study. We were not able to examine the effect of the level of inbreeding among individual females on possible maternal traits such as clutch size (cf. van Noordwijk and Scharloo, 1981; but see Gibbs [1988]) because only three inbred females reproduced during our study (see below).

\section{RESULTS}

\section{Matings Between Related Birds}

Table 1 shows observed levels of inbreeding (percentage matings between related individuals) for different subsets of all unique pairings between different males and different females. In the period 1981-1987, 4.6\% ( 27 of 583) of all pairings were between related individuals, with coefficients of kinship ranging from 0.008 to 0.250 . The observed distribution of $\phi$ among inbred matings was: $0.008(N=1$ pair $), 0.016(N$ $=3), 0.031(N=3), 0.047(N=1), 0.063$ $(N=10), 0.094(N=1), 0.125(N=5)$, and $0.250(N=3)$. Matings between distant kin $(\phi<0.125)$ make an important numerical contribution to the overall levels of inbreeding in this population; more than $70 \%$ of all inbred matings are between distant kin, and these matings contribute $40.1 \%$ to the summed values of $\phi$ for all pairings.

As emphasized by van Noordwijk and
Scharloo (1981), observed levels of inbreeding are strongly influenced by the extent of our knowledge of the ancestry of individuals in a population. The positive effect of pedigree depth on detected inbreeding is clear in these data. Detected matings between related birds increased from $4.6 \%$ for all pairs to more than $20 \%$ for pairs for which at least half of the grandparents were known (Table 1). The comparable estimates of mean pair relatedness are 0.004 and 0.012 , respectively. Detected levels of close inbreeding ( $\phi \geq 0.125$ ), often cited as a measure of population levels of inbreeding (cf. Greenwood et al., 1978), ranged from $0 \%$ to $2 \%$ for all pairs in different categories. Thus, on average, for birds with at least half of their grandparents known, a minimum of $20 \%$ of their matings are between related birds, resulting in a minimum mean $\phi$ value of 0.01 for all pairs.

\section{Effect of Relatedness on Pairing}

To see whether pairing was nonrandom with respect to relatedness, we compared observed levels of inbreeding with those generated from simulated episodes of pairing in which mating between birds was random with respect to relatedness. Figure 1 compares the distributions of mean relatedness per episode of random mating (for 1,000 episodes of pairing) with the observed value for five separate breeding periods. There is a clear effect of pedigree depth on both the continuity and symmetry of the random distributions. For 1981 , the distribution is strongly skewed to the right, and there are frequent gaps along the abcissa. By 1987 the center of the distribution had shifted to the right, reflecting the increase in detected levels of inbreeding, although the distribution itself was still skewed to the right. The lack of low relatedness values in certain 

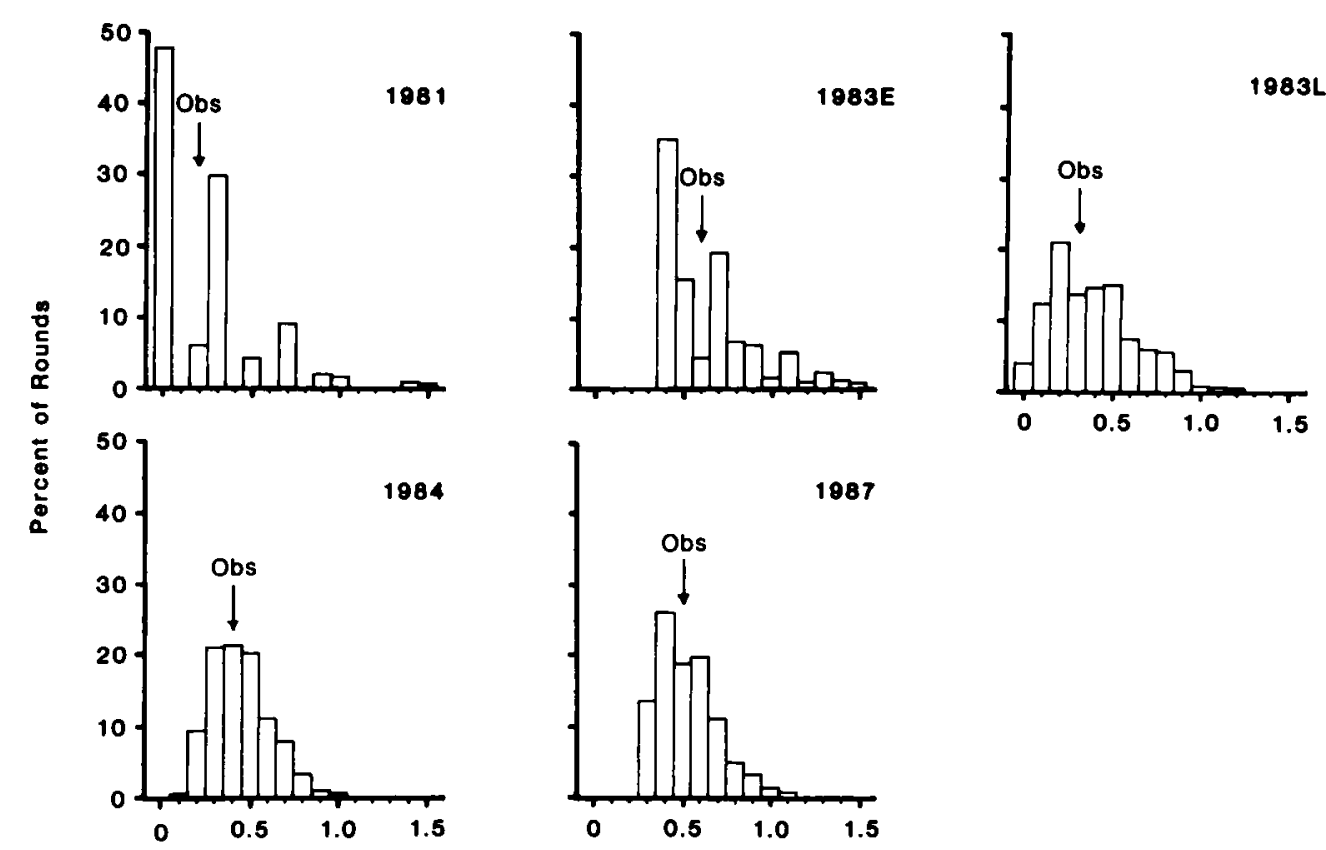

Mean Relatedness Per Round of Random Mating $\left(\times 10^{2}\right)$

FIG. 1. Distributions of mean inbreeding per round of pairing for simulations of random mating by $G$. fortis with respect to relatedness. Five breeding episodes are shown, which simulate pairing during the first round of breeding in 1981, 1983 (1983E), 1984, and 1987 and for an episode of pairing in the middle of the 1983 breeding season (1983L; see Materials and Methods). Observed levels of inbreeding are indicated by arrows above each distribution.

periods (e.g., 1983E) as compared to others (e.g., 1981 and 1983L) is due to the varying persistence of small numbers of long-term inbred pairs from one year to the next. For each breeding episode, however, the observed level of inbreeding (mean relatedness of first pairings by each member of the limiting sex in each episode; see Materials and Methods) is very close to the middle value of each of the random distributions.

Table 2 gives a quantitative description of how well random mating predicts observed inbreeding in each breeding episode. For each episode, the difference between the median value of $\phi$ from the random distribution and the observed value of $\phi$ is extremely small: the maximum difference is 0.021 , or less than $7 \%$ of the observed value of $\phi$. The fact that, in each case, almost exactly half of the random simulations (range: 48-56\%) have values less than the observed value also demonstrates the close correspondence between the expected random values and the observed values of $\phi$. Thus, in all years, the observed values of inbreeding appear to be extremely close to those expected if pairing between individual birds were random with respect to relatedness.

The use of population-wide estimates of pairing (i.e., mean relatedness per round of pairing for all birds) could mask subtle effects of relatedness on pairing. For example,

TABLE 2. Expected versus observed levels of inbreeding in $G$. fortis from 1981 to 1987. All values for $\phi$ have been multiplied by 100 . Only the first breeding episode is used for 1981, 1984, and 1987 (see text).

\begin{tabular}{|c|c|c|c|c|}
\hline Year & $\begin{array}{l}\text { Median } \phi \\
\text { from random } \\
\text { distribution }\end{array}$ & Observed $\phi$ & $\begin{array}{l}\text { Percentage of } \\
\text { simulations } \\
\text { with values } \\
\text { less than } \\
\text { observed }\end{array}$ & $\begin{array}{l}\text { Observed } \\
\text { number of } \\
\text { pairs per } \\
\text { breeding } \\
\text { episode } \\
\text { (number of } \\
\text { related pairs) }\end{array}$ \\
\hline 1981 & 0.176 & 0.164 & 49.0 & $76(1)$ \\
\hline \multicolumn{5}{|l|}{1983} \\
\hline $\begin{array}{l}\text { Early } \\
\text { Late }\end{array}$ & $\begin{array}{l}0.536 \\
0.335\end{array}$ & $\begin{array}{l}0.554 \\
0.314\end{array}$ & $\begin{array}{l}55.4 \\
47.9\end{array}$ & $\begin{array}{r}79(3) \\
185(7)\end{array}$ \\
\hline 1984 & 0.432 & 0.440 & 51.7 & $164(8)$ \\
\hline 1987 & 0.508 & 0.510 & 52.6 & $93(4)$ \\
\hline
\end{tabular}


TABLE 3. Expected and observed frequencies of related matings among newly formed pairs in each year. Expected values were calculated from simulations for each breeding episode and only include pairings with $\phi$ values greater than zero.

\begin{tabular}{|c|c|c|c|c|c|c|c|c|}
\hline \multirow[b]{4}{*}{ Year } & \multicolumn{8}{|c|}{ Relatedness } \\
\hline & \multicolumn{4}{|c|}{$\phi<0.125$} & \multicolumn{4}{|c|}{$\phi \geq 0.125$} \\
\hline & \multicolumn{2}{|c|}{ Expected } & \multicolumn{2}{|c|}{ Observed } & \multicolumn{2}{|c|}{ Expected } & \multicolumn{2}{|c|}{ Observed } \\
\hline & $N$ & $\%$ & $N$ & $\%$ & $N$ & $\%$ & $N$ & $\%$ \\
\hline 1981 & 0 & 0 & 0 & 0 & 92 & 100 & 1 & 100 \\
\hline \multicolumn{9}{|l|}{1983} \\
\hline $\begin{array}{l}\text { Early } \\
\text { Late }\end{array}$ & $\begin{array}{r}34 \\
376\end{array}$ & $\begin{array}{l}21 \\
60\end{array}$ & $\begin{array}{l}1 \\
3\end{array}$ & $\begin{array}{l}50 \\
50\end{array}$ & $\begin{array}{l}127 \\
247\end{array}$ & $\begin{array}{l}79 \\
40\end{array}$ & $\begin{array}{l}1 \\
3\end{array}$ & $\begin{array}{l}50 \\
50\end{array}$ \\
\hline 1984 & 679 & 68 & 5 & 83 & 320 & 32 & 1 & 17 \\
\hline 1987 & 214 & 75 & 9 & 90 & 70 & 25 & 1 & 10 \\
\hline
\end{tabular}

if avoidance of pairing with close kin is offset by preferential pairing with more distant kin (see Bateson, 1978), then the mean kinship coefficient, averaged across all pairs, would be similar to one calculated if pairing were random. To see whether such a subtle pattern of mate choice occurred, for the simulations for each breeding episode we determined all possible pairings between related individuals and calculated the numbers of pairs in two categories of relatedness, those with kinship coefficients of at least 0.125 and those with coefficients less than 0.125 but greater than zero. We then compared these numbers of pairs with similar values for observed newly formed pairings between related birds in each period. Sam- ple sizes are small, making statistical tests inappropriate, but in no case is there strong evidence that close kin are being avoided while more distant kin are preferred (Table $3)$.

\section{Reproductive Success of Related Pairs}

To determine whether inbreeding affects reproductive success, we compared the observed success of related pairs with estimates of expected success based on the performance of pairs not known to be related (Table 4). A paired comparison of observed with expected values for all related pairs (mean $\phi=0.085 ; N=27$ ) shows no significant differences for eight components of reproductive success.

TABLE 4. Expected and observed measures of reproductive success for related pairs of $G$. fortis. $P$ values are from a paired $t$ test. MSD is the smallest difference between expected and observed that can be detected for a given sample using a two-tailed $t$ test with a 0.05 level of significance (Zar, 1984). The percentage of young that fledged was measured only for matings from which at least one egg hatched; the percentage of young that survived was measured only for matings from which at least one offspring fledged.

\begin{tabular}{clcccccc}
\hline \hline Types of pairs & \multicolumn{1}{c}{ Measure of success } & $N$ & Expected & Observed & $t$ & $P$ & MSD \\
\hline All related & Clutch size & 27 & 3.49 & 3.50 & 0.06 & 0.95 & 0.30 \\
$(\phi>0)$ & Number of eggs hatched & 27 & 2.01 & 1.65 & 1.64 & 0.11 & 0.45 \\
& Number of young fledged & 27 & 1.67 & 1.40 & 1.28 & 0.21 & 0.43 \\
& Number of young survived & 16 & $\mathbf{0 . 6 9}$ & 0.52 & 1.09 & 0.29 & 0.34 \\
& Percentage of eggs hatched & 27 & 54 & 45 & 1.74 & 0.09 & 11 \\
& Percentage of young fledged & 21 & 79 & 85 & 0.90 & 0.38 & 13 \\
& Percentage of eggs fledged & 27 & 45 & 38 & 1.38 & 0.18 & 11 \\
& Percentage of young survived & 14 & 48 & 33 & 1.58 & 0.14 & 21 \\
Closely related & Clutch size & 8 & 3.57 & 3.34 & 0.69 & 0.51 & 0.77 \\
$(\phi \geq 0.125)$ & Number of eggs hatched & 8 & 1.92 & 1.68 & 0.52 & 0.62 & 1.12 \\
& Number of young fledged & 8 & 1.58 & 1.52 & 0.18 & 0.90 & 1.07 \\
& Number of young survived & 6 & 0.77 & 0.72 & 0.12 & 0.91 & 0.99 \\
& Percentage of eggs hatched & 8 & 51 & 44 & 0.77 & 0.46 & 22 \\
& Percentage of young fledged & 6 & 79 & 75 & 0.40 & 0.71 & 25 \\
& Percentage of eggs fledged & 8 & 41 & 36 & 0.51 & 0.62 & 22 \\
& Percentage of young survived & 5 & 51 & 39 & 1.11 & 0.33 & 30 \\
\hline
\end{tabular}




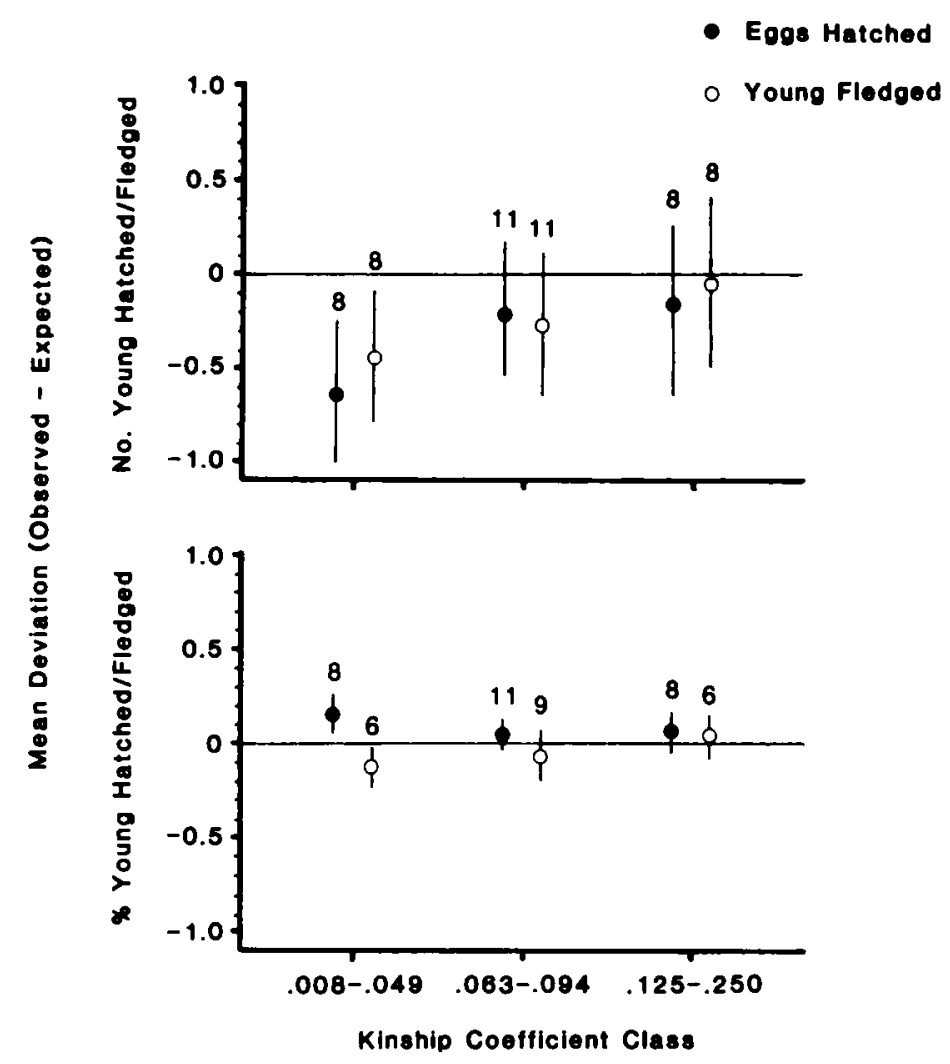

FIG. 2. The relationship between average deviations (observed success - expected success) for different measures of reproductive success and the coefficient of kinship for related pairs of $G$. fortis. The measures of success were: number of eggs hatched and number of young fledged (above), and percentage of eggs hatched and percentage of hatched young that fledged (below). Values are means \pm SE. Sample sizes (numbers of pairs) are given above each point.

To determine whether significant differences in reproductive success occur only among closely related ( $\phi \geq 0.125$ ) pairs (see Greenwood et al., 1978; Ralls et al., 1986), we repeated the above analyses using only pairs with a $\phi$ value of 0.125 or greater (Table 4). As before, there was no detected difference between observed and expected values of reproductive success for related pairs, although sample sizes are small $(N=5-8)$ and the values for the minimum detectable significant difference (MSD) show that the power of the statistical tests is low.

If inbreeding depression occurred, the magnitude of this depression should covary positively with pair relatedness; we would therefore expect a significant correlation between $\phi$ and the difference between expected and observed values of reproductive success. There is no such relationship for four measures of reproductive success (Fig. 2). The upper part of the figure shows that the depression is, in fact, greatest for the least related class, not for the most related one. Rank correlations between the differences in each of the eight measures of reproductive success in Table 4 and $\phi$ ranged between -0.29 and 0.05 . None of the differences was significant, and the signs of the correlations varied, indicating that there was no consistent relationship between degree of inbreeding and success.

\section{Inbred Offspring}

Inbred offspring are both viable and fertile. A total of eight inbred offspring, produced from matings between related parents in early 1983 , were recruited into the breeding population from late 1983 to 1987 . They consisted of five males, including four brothers from the same clutch and three females, each from different nests. All the brothers had coefficients of inbreeding $(F)$ 
of 0.125 , while the fifth male had an $F$ value of 0.063 ; two of the females had $F=0.125$, while the other had an $F=0.063$. All these birds had at least one nest, and their clutch sizes ranged from two to five eggs. One of the male siblings had a total of six nests, while two of the inbred females $(F=0.125$ and 0.063 ) had seven nests each. Six of the eight birds fledged at least one young.

\section{Discussion}

There are still relatively few studies of inbreeding in birds based on pedigree analysis (for review see Ralls et al. [1986]). Nonetheless, the work done so far suggests that observed levels of inbreeding are comparable among species with similar social behavior. As shown in this study, levels of inbreeding appear to be determined more by population structure than by behavioral avoidance of mating with relatives (see also van Noordwijk et al. [1985] and van Tienderen and van Noordwijk [1988]). In contrast, the effects of inbreeding on reproductive success differ considerably between populations.

\section{Levels of Inbreeding}

To compare the frequency of inbreeding in different populations, it is necessary to take into account the differences in pedigree depth. This can be done in two related ways: 1) by estimating a mean $\phi$ for a sample of birds with a certain minimum number of known ancestors and 2) by estimating the percentage of all matings made up of pairings between particular classes of relatives. To our knowledge, van Noordwijk and Scharloo (1981) presented the only previous estimate of inbreeding based on the first method. They estimated the mean value of $\phi$ to be 0.036 for the Vlieland population of Great Tits, based on pairs for which at least grandparents were known. The equivalent value for $G$. fortis is 0.011 (Table 1), suggesting that inbreeding may be somewhat lower in the finches. However, the estimate for $G$. fortis is much less reliable, because it is based on a much smaller sample size: all grandparents were known for $33 \%$ of all Great Tit pairs, but for only $2 \%$ of all Darwin's Medium Ground Finch pairs.

The percentage of all matings between very closely related birds ( $\phi \geq 0.250$ ) pro- vides a less complete but more widely available comparative measure of the levels of inbreeding in populations. Ralls et al. (1986) summarize the available estimates for native birds that do not breed cooperatively, using mostly unpublished data. The percentage for five mainland and one island population of four species ranges from $0.4 \%$ for the Pied Flycatcher (Ficedula hypoleuca) to $3.0 \%$ for the Vlieland Great Tit population. The equivalent figure for $G$. fortis on Daphne is $0.6 \%$. Thus, inbreeding among $G$. fortis on Daphne appears to be as frequent as in some other species with similar social behavior but somewhat rarer than in the Great Tit.

The consistently low percentages of closely inbred matings in these populations suggested to Ralls et al. (1986) that close inbreeding may be avoided behaviorally. This appears unlikely to us, since pairing occurs at random with respect to relatedness in two species, Darwin's Medium Ground Finches and Great Tits, which span the range $(0.6 \%$ $3.0 \%$ ) in terms of percentages of closely inbred pairs. More likely, the similarity in levels of inbreeding between studies is a result of similarity in the effective population sizes of the birds under study (van Tienderen and van Noordwijk, 1988). We emphasize, as Ralls et al. (1986) acknowledge, that inbreeding avoidance can only be directly demonstrated by comparing the observed pattern of mating with a random expectation.

\section{Effect of Inbreeding}

The major difference between this study and the detailed work on Great Tits (Greenwood et al., 1978; van Noordwijk and Scharloo, 1981) is that we could detect no effect of inbreeding on reproductive success, whereas van Noordwijk and Scharloo (1981) detected a strong and significant effect, mainly on hatching success. Our result is not without precedent. Rowley et al. (1986) recently reported that reproductive success did not differ between very closely related $(\phi \geq 0.250)$ and less closely related $(\phi<$ $0.250)$ pairs of Splendid Fairy Wrens ( $\mathrm{Ma}$ lurus splendens) (see also Payne et al. [1985], James et al. [1987], and Payne et al. [1987]).

Clearly, our sample sizes of related pairs of finches were small, particularly the num- 
ber of closely related pairs $(\phi \geq 0.125)$, in which possible costs of inbreeding are expected to be highest. Our analyses of success thus have a conservative bias because some pairs of birds that we assumed to be unrelated may in fact share recent common ancestors. Therefore, more data are needed to confirm the conclusion that costs of inbreeding are small or nonexistent in these birds. Nevertheless, based on the argument presented below, we suggest that, even if additional data were to result in the detection of a cost, its magnitude would probably still be substantially lower than the cost of inbreeding observed in Great Tits.

If, as in Great Tits, the negative effects of inbreeding act mainly to reduce hatching success and if the magnitude of this effect can be estimated as the difference in success between related and nonrelated pairs, then we can compare the potential cost of inbreeding in $G$. fortis with the demonstrated cost in Great Tits. Restricting the analysis to closely related pairs $(\phi \geq 0.125)$, the reduction in the number of eggs hatched is approximately 1.25 for pairs of Great Tits and 0.24 for pairs of $G$. fortis. Part of the difference can be explained by the larger mean clutch size of Great Tits, which is about twice that of $G$. fortis (van Noordwijk et al., 1981). Nevertheless, on a percentage basis, the loss in eggs hatched is $18 \%$ in Great Tits and only $7 \%$ in $G$. fortis (values for Great Tits were estimated from fig. 4 and table 5 in van Noordwijk and Scharloo [1981]; those for $G$. fortis were based on the data in Table 4). The loss for the finches appears to be substantially lower than the loss for Great Tits; however, the small nonsignificant increase in recruitment for inbred Great Tit young relative to outbred young may reduce this difference slightly (van Noordwijk and Scharloo, 1981).

Even though present levels of inbreeding are similar, the difference between Great Tits and $G$. fortis in the effect of inbreeding on reproductive success may be due to historical differences in population size and structure that have led to reduced levels of heterozygosity and lower numbers of deleterious alleles in the $G$. fortis population. The Great Tit population on Vlieland appears to be relatively open and outbred: over the study period, $8.5-30.8 \%$ of breed- ing males and $20.3-49.1 \%$ of breeding females (depending on area) were presumably nonrelated immigrants (van Tienderen and van Noordwijk, 1988). In contrast, the Daphne population of $G$. fortis is much more closed, with a maximum of $0-3 \%$ immigrants per breeding season. Although some hybridization also occurs (see Gibbs and Grant, 1987a), it is infrequent (approximately $2 \%$ per breeding season). Severe population bottlenecks on Daphne can also occur, with the number of breeding females ranging from more than 200 birds to 28 individuals in one year (Price et al., 1984). These factors probably combine to reduce the long-term effective population size of $G$. fortis to a level much lower than that of the Vlieland Great Tit population. Thus, deleterious alleles may have been purged from the finch population in the past (see Templeton, 1987), accounting for the difference in the effects of inbreeding on reproductive success between the two species. This hypothesis could be indirectly tested by comparing current levels of heterozygosity in the G. fortis and Great Tit populations using genetic markers (cf. Yang and Patton, 1981). The prediction is that the $G$. fortis population should have lower levels of variation than the Great Tit population (Nei et al., 1975).

\section{Avoidance of Inbreeding?}

There is behavioral evidence that close inbreeding is avoided in some cooperatively breeding birds (e.g., Koenig and Pitelka, 1979), even though the supposed reason for such behavior (a cost to inbreeding) has not been demonstrated. Nonetheless, the close correspondence between observed levels of inbreeding and those predicted under a model of random pairing with regard to relatedness strongly suggests that population structure is the primary determinant of observed levels of inbreeding in the $G$. fortis population on Isla Daphne Major. Similarly, van Noordwijk et al. (1985) and van Tienderen and van Noordwijk (1988) found little difference between the mean kinship coefficients of observed and randomly generated pairs of Great Tits. These studies of the Great Tit and our study of $G$. fortis are the only ones that have generated expected levels of inbreeding under models of ran- 
dom pairing and then compared the results with observations from a wild population.

The lack of discrimination against mating with relatives does not appear to be related to the presence or absence of a cost to inbreeding. Both Great Tits, which show a cost, and $G$. fortis, for which there is no evidence of a cost, mate at random with relatives. It is likely that factors other than kinship have a stronger effect on mate choice in both species. In $G$. fortis, these factors include the size and age of the birds and territory size (Price, 1984). In $G$. conirostris on Isla Genovesa, age-related experience and behavior of males is of overriding importance in attracting a mate (Grant and Grant, 1987). Another possibility is that kin recognition is poorly developed in all these species. In birds, kin recognition appears to develop through social association, rather than through direct assessment of genetic relatedness (Beecher, 1982; Payne et al., 1988). Thus, while parents or nestmates could recognize each other, the opportunity to develop recognition of more distant relatives or siblings born at different times may be absent (Grant, 1984).

To conclude, while levels of inbreeding are comparable to those found in other passerines, there was no detectable effect of inbreeding on reproductive success, or of relatedness on mate choice in $G$. fortis. Population structure seems to be a more important determinant of levels of inbreeding in these birds than does behavioral avoidance of mating with kin.

\section{ACKNOWLEDGMENTS}

We thank P. Boag, M. Bulmer, P. Myers, B. Grant, R. Payne, T. Price, P. van Tienderen, and especially P. Smouse for comments and discussion; G. Keyes, S. Latta, D. McCullough, and J. Weiland, for help with the field work; R. Bush and J. Tworek for assistance with the analysis; and T. Price for the use of his data. This work was carried out with the permission of the Direccion General de Desarrollo, Quito, and with the help of the Servicio Parque Nacional Galápagos, and the Charles Darwin Research Station. It was supported by NSF grants to P.R.G. H.L.G. was supported by an NSERC (Canada) Postgraduate Scholarship, Block Grants from the Department of
Biology, University of Michigan, and a Hinsdale-Walker Scholarship from the Museum of Zoology, University of Michigan.

\section{Literature Cited}

BAteson, P. P. G. 1978. Sexual imprinting and optimal outbreeding. Nature 273:659-660.

BEECHER, M. D. 1982. Signature systems and kin recognition. Amer. Zool. 23:477-490.

BOAG, P. T., AND P. R. GRant. 1984. Darwin's Finches (Geospiza) on Isla Daphne Major, Galápagos: Breeding and feeding ecology in a climatically variable environment. Ecol. Monogr. 54:463489.

BULMER, M. G. 1973. Inbreeding in the Great Tit. Heredity 30:313-325.

CRAIG, J. L., AND I. JAMIESON. 1988. Incestuous mating in a communal bird: A family affair. Amer. Natur. 131:58-70.

CROW, J. F., AND M. Kimura. 1970. An Introduction to Population Genetic Theory. Harper and Row, N.Y.

Daniell, A., And N. D. Murray. 1986. Effects of inbreeding in the Budgerigar (Melopsittacus undulatus: Aves: Psittacidae). Zoo Biol. 5:233-238.

GIBBS, H. L. 1988. Heritability and selection on clutch size in Darwin's Medium Ground Finches (Geospiza fortis). Evolution 42:750-762.

GibBs, H. L., AND P. R. Grant. 1987a. Ecological consequences of an exceptionally strong El Niño event on Darwin's Finches. Ecology 68:1735-1746. $1987 \mathrm{~b}$. Adult survivorship in Darwin's Ground Finch populations in a variable environment. J. Anim. Ecol. 56:797-813.

Gibis, H. L.. P. R. Grant, and J. Weiland. 1984. Breeding of Darwin's Finches at an exceptionally early age in an El Niño year. Auk 101:872-874.

GRANT, B. R. 1984. The significance of song variation in a population of Darwin's Finches. Behavior 89 : 90-116.

Grant, B. R., AND P. R. Grant. 1987. Mate choice in Darwin's Finches. Biol. J. Linn. Soc. 32:247270.

Greenwood, P. J., and P. H. Harvey. 1982. The natal and breeding dispersal of birds. Ann. Rev. Ecol. Syst. 13:1-21.

Greenwood, P. J., P. H. Harvey, and C. M. Perrins. 1978. Inbreeding and dispersal in the Great Tit. Nature 271:52-54.

James, P. C., L. Q. Oliphant, and I. G. WARkentin. 1987. Close inbreeding in the Merlin (Falco columbarius). Wilson Bull. 99:718-719.

Koenig, W. D., and F. A. Pitelka. 1979. Relatedness and inbreeding avoidance in the communally nesting Acorn Woodpecker. Science 206:1 103-1 105.

LERNER, I. M. 1954. Genetic Homeostasis. Wiley, N.Y.

Malécot, G. 1948. Les Mathématiques de l'Hérédité. Masson, Paris, France.

MAYR, E. 1963. Animal Species and Evolution. Harvard Univ. Press, Cambridge, MA.

Nei, M., T. Maruyama, ANd R. Chakraborty. 1975. The bottleneck effect and genetic variability in populations. Evolution 29:1-10. 
Payne, R. B., L. L. Payne, and S. M. Doehlert. 1987. Song, mate choice, and the question of kin recognition in a migratory song bird. Anim. Behav. $35: 35-47$.

Payne, R. B., L. L. Payne, and I. Rowley. 1985. Splendid wren Malurus splendens response to cuckoos: An experimental test of social organization in a communal bird. Behaviour 94:108-127.

. 1988. Kinship and nest defense in co-operative birds: Splendid Fairy Wrens (Malurus splendens). Anim. Behav. 36:939-941.

Price, T. D. 1984. Sexual selection and body size, plumage, and territory variables in a population of Darwin's Finches. Evolution 38:327-341.

- 1985. Reproductive responses to varying food supply in a population of Darwin's Finches: Clutch size, growth rates, and hatching synchrony. Oecologica $66: 411-416$.

Price, T. D., P. R. Grant, and P. T. Boag. 1984. Genetic changes in the morphological differentiation of Darwin's Ground Finches, pp. 49-66. In K. Wohrmann and V. Loescheke (eds.), Population Biology and Evolution. Springer-Verlag, Berlin, W. Ger.

Ralls, K., P. H. HaRvey, AND A. M. Lyles. 1986. Inbreeding in natural populations of birds and mammals, pp. 35-56. In M. Soulé (ed.), Conservation Biology: The Science of Scarcity and Diversity. Sinauer, Sunderland, MA.

Reinhardt, P. S. (ed.). 1979. SAS Supplemental User's Guide. SAS Inst., Inc., Cary, NC.

Rowley, I., E. Russell, AND M. BroOker. 1986. Inbreeding: Benefits outweigh costs. Anim. Behav. 34:939-941.

Shields, W. M. 1982. Philopatry, Inbreeding, and the Evolution of Sex. State Univ. New York Press, Albany, NY.
ShoffNer, R. N. 1948. The reaction of fowl to inbreeding. Poultry Sci. 27:448-452.

Sittmann, K., H. Abplanalp, and R. A. Fraser. 1966. Inbreeding depression in Japanese Quail. Genetics 54:371-379.

TEMPLETON, A. J. 1987. Inferences on natural population structure from genetic studies on captive mammalian populations, pp. 257-272. In B. D. Chepko-Slade and Z. T. Halpin (eds.), Mammalian Dispersal Patterns. Univ. Chicago Press, Chicago, IL.

van NoOrdwuk, A. J., AND W. Scharloo. 1981. Inbreeding in an island population of the Great Tit. Evolution 35:674-688.

van NoOrdwik, A. J., J. H. van Balen, and W. SCHARLOO. 1981. Genetic and environmental components of variation in clutch size of the Great Tit (Parus major). Neth. J. Zool. 31:342-373.

van Noordwuk, A. J., P. H. van Tienderen, G. DE JoNG, AND J. H. VAN BALEN. 1985. Geneological evidence for random mating in a natural population of the Great Tit (Parus major L.). Naturwissenshaften 72:104-106.

VAN TIEnderen, P. H., AND A. J. VAN NoORdWiJk. 1988. Dispersal, kinship and inbreeding in an island population of the Great Tit. J. Evol. Biol. 1: 117-137.

WRIGHT, S. 1969. Evolution and the Genetics of Populations, Vol. 2. The Theory of Gene Frequencies. Univ. Chicago Press, Chicago, IL.

YANG, S. Y., AND J. L. PAtton. 1981. Genic variability and differentiation in Galapagos finches. Auk 98:230-242.

ZAR, J. H. 1984. Biostatistical Analysis, 2nd Ed. Prentice-Hall, Englewood Cliffs, NJ.

Corresponding Editor: G. de Jong 